\title{
An Easy Way for Percutaneous Nephrolithotomy Using Mixed Sedative Agents
}

\author{
Amr M. Hilal Abdou*, Walid Y. Kamel, Eman K. Abo-Seif \\ Department of Anesthesia, Intensive Care, and Pain Management, Ain Shams University, Cairo, Egypt \\ Email: *amr_hilal@med.asu.edu.eg
}

How to cite this paper: Abdou, A.M.H., Kamel, W.Y. and Abo-Seif, E.K. (2018) An Easy Way for Percutaneous Nephrolithotomy Using Mixed Sedative Agents. Open Journal of Anesthesiology, 8, 205-213. https://doi.org/10.4236/ojanes.2018.87021

Received: April 27, 2018

Accepted: July 22, 2018

Published: July 25, 2018

Copyright $\odot 2018$ by authors and Scientific Research Publishing Inc. This work is licensed under the Creative Commons Attribution International License (CC BY 4.0).

http://creativecommons.org/licenses/by/4.0/

\begin{abstract}
Objective: We recorded the data of patients performing Percutaneous Nephrolithotomy (PCNL) under combined spinal anesthesia with sedative mixtures of Ketamine-Propofol (KP) or Fentanyl-Propofol (FP). Background: The PCNL is usually done under General anesthesia (GA); combining spinal anesthesia with a mixture of sedative drugs has shown to provide an optimum intra and post-operative analgesic and sedative response without side effects. Patients and Methods: 100 healthy patients were enrolled for PCNL, after stabilization of the sensory block of spinal anesthesia patients were randomized into two groups; Group KP received $1 \mathrm{mg} / \mathrm{Kg}$ Ketamine and $1 \mathrm{mg} / \mathrm{Kg}$ Propofol diluted in $20 \mathrm{ml}$ syringe given over 30 seconds and Group FP received $1 \mathrm{mic} / \mathrm{Kg}$ Fentanyl and $1 \mathrm{mg} / \mathrm{Kg}$ Propofol diluted in $20 \mathrm{ml}$ syringe over 30 seconds; both groups will receive increment doses if the patient suffers from anxiety, pain or discomfort. Perioperative Hemodynamic data (HR, SBP, $\mathrm{DBP}, \mathrm{RR}$, and SpO2) were recorded; PACU stay time and post operative analgesia time were analyzed. Results: Sedative mixtures of FP and KP provided remarkably deep sedation levels for PCNL procedures under spinal anesthesia. Respiratory depression, hypotension and bradycardia were the major side effects reported in FP group which had mean decrease in RR of 4.63 breath/min $(27.49 \%)$ with mean $3.77 \%$ drop in SpO2 levels. KP group produced significant increase in HR about 7 beats/min without reported side effects. Conclusion: Various sedation techniques may be applicable for PCNL procedures with routine premedication, mixtures of fentanyl-Propofol or Ketamine-Propofol could be used, and KP has advantages of stable hemodynamics, prolonged analgesic response intra and post-operatively with no appreciable side effects and more effective method of sedative mixture.
\end{abstract}

\section{Keywords}

Nephrolithotomy, Percutaneous, Sedation, Propofol, Ketamine 


\section{Introduction}

There has been an increased use of Percutaneous Nephrolithotomy (PCNL) nowadays as being the gold standard procedure for treatment of difficult, large $\geq 2$ $\mathrm{cm}$, and staghorn renal stones [1]. PCNL is usually a difficult and lengthy procedure requiring an adequate access to the kidneys and the stone besides identifying the optimum tract for stone retrieval [2]. Anesthetic decision for PCNL is based on the surgical anatomy of the kidneys which lies retroperitoneal with a significant portion of it lies subcostally, specially the lower pole rendering the upper pole in an higher level causing intraoperative irritation and pain during the procedure. Regional anesthesia is popular and offers several benefits to the patients. The top three advantages from the patient's point of view are staying awake, early family contact, and early food intake. For the anesthetist, cardiovascular and respiratory stability, rapid postoperative recovery, and preservation of protective airway reflexes are the most important advantages of regional anesthesia [3], and despite the potency and efficacy of intrathecal anesthesia for Lower calyceal stones, some upper pole renal stones are usually spared from the effect of regional anesthesia which needs further anesthetic requirements. This ignites the idea of adding a sedative method in addition to a conventional intrathecal anesthesia to prevent the above mentioned drawbacks such as pain and irritation. Goals of sedation include providing an adequate level of sedation while minimizing pain and anxiety, maximizing amnesia, minimizing the potential for adverse drug-related events, controlling behavior, and maintaining a stable cardiovascular and respiratory status [4]. Several drugs has been used to accomplish all of these goals depending on the onset, duration, safety, and financial availability, still at this time in Egypt there is no single agent unique as a sedative agent during PCNL in prone position, therefore many anesthesiologists usually combine different drugs to achieve a desired satisfactory goal [5]. It is believed that combined spinal anesthesia with sedation can reduce the anesthetic requirements; peak sedation effect is usually detected 30 - 45 minutes after the block [6] [7]. In the setting of regional anesthesia, hypnosis has been used to provide light sedation and amnesia. However, success of this technique was limited by the need of supplementary analgesics. As the patient needs to relax and concentrate for the induction of hypnosis, most attempts for emergency operations failed and in elective cases for more than one hour. In the hands of an experienced specialist, hypnosis may be useful in suitable patients, and when sedatives are contraindicated [8]. Various sedatives techniques have been suggested to achieve a satisfactory level of sedation depending on blood level along with the hemodynamic effects of the drugs [9]. Midazolam has suitable properties for premedication as it is anxiolytic, provides good amnesia, decreases Propofol (PROP) requirements, but does not prolong the recovery roomstay [10]. Then clonidine started to participate as a good anxiolytic decreasing the PROP requirements but without amnesia [11], another agent discovered in 1960 is the Ketamine (KT) which is a good analgesic with weak hypnotic property [12]. Ketamine, Midazo- 
lam, Propofol and also opioids have been used in a variety of combination, particularly during Total intravenous anesthesia (TIVA). Ketamine in relatively low doses is arguably the safest agent currently available for the induction of anesthesia but it has been undervalued due to dysphoric phenomena during recovery and its relatively prolonged duration of action when used as sole agent. The aim of this study is to find and evaluate a unique sedation regimen for PCNL procedures using a combination of either Ketamine-Propofol (KT-PROP) or Fentanyl-Propofol (FT-PROP) mixtures as an adjuvant to spinal anesthesia for these procedures.

\section{Sample Size}

Using PASS program, setting alpha error at 5\% and power $80 \%$. Result from previous study [13] Based on this the needed sample we recruited 100 cases for the study.

\section{Methodology}

The ethical committee of faculty of medicine, Ain Shams University, Cairo approved a feasibility study in 100 patients after giving a written informed consent for PCNL and the study, these healthy ASA 1 patients aged 20 - 40 years old diagnosed of renal stone more than $2 \mathrm{~mm}$ candidate for disintegration were randomly allocated into two groups; Group I : Ketamine-Propofol (KP) group and Group II : Fentanyl-Propofol (FP) group in addition to conventional spinal anesthesia, patients with morbid obesity BMI $\geq 30$, mental retardation, history of substance abuse or drug allergy were excluded from the study. A preoperative visit is done to justify and clarify that any pain, discomfort or anxiety during the procedure in prone position will be dealt pharmacologically immediately, also the urology surgical team were instructed to ask for general anesthesia if they felt any technical difficulty during the procedure. All patient were monitored using standard technique throughout the procedure, Spinal anesthesia was done using hyperbaric bupivacaine $0.5 \% 2.5 \mathrm{ml}$, satisfactory sensory block assessed every 3 minutes for the first 15 minutes till stabilization of the level of the block, then Group I (KP) received a fresh mixture of $1 \mathrm{mg} / \mathrm{Kg}$ Ketamine and $1 \mathrm{mg} / \mathrm{Kg}$ Propofol diluted in $5 \%$ dextrose solution in $20 \mathrm{ml}$ syringe given intravenously over 30 seconds as a bolus dose followed by increments if the patients complaint of any pain or discomfort, the second group II (FP) received a mixture of $1 \mathrm{mic} / \mathrm{Kg}$ Fentanyk and $1 \mathrm{mg} / \mathrm{Kg}$ Propofol diluted in 5\% dextrose also as a bolus followed by increments. During the procedure supplemental oxygen of $4 \mathrm{~L} / \mathrm{min}$ was given via a nasal cannula if $\mathrm{SpO} 2$ was $\leq 90 \%$, The technique of PCNL was done by inserting a guide wire in the collecting system, then passing a nephroscope alongside the guide wire then the disintegration process started, if the patient feels discomfort or pain (VAS $\geq 3$ ) increments of $\mathrm{KP}$ or FP are given, if the problem is not resolved GA is started after turning the patient into supine position with Endotracheal intubation and the case is reported as a failed technique. During 
the procedure we assessed the hemodynamic parameters (HR, SBP, DPB, RR, SpO2) recorded every 1 minute for the first 10 minutes then every 10 minutes. Any hemodynamic instability will be dealt via atropine or ephedrine and fluids. In the PACU; vital data were recorded every 15 minutes in addition to the time of PACU stay. Postoperative pain was assessed for the first six hours using the VAS at the following times $0 \mathrm{~h}, 1 \mathrm{~h}, 3 \mathrm{~h}$ and $6 \mathrm{~h}$.

\section{Statistical Analysis}

The study was double-blinded with three different anesthesiologists involved. The drugs were prepared by an anesthesia resident not involved in the study and were blinded to the study groups. The anesthesiologist who pretreated the patients was blinded to each patient's allocation. The study drug solutions were identical in appearance. Data were analyzed using Chi-square test for categorical data. Student's $t$-test for quantitative variables for comparison between the two groups. For intra group comparison, paired $t$-test was used. Results were expressed as mean \pm SD. Probability value less than 0.05 was considered significant.

\section{Results}

Hundred patients were enrolled for PCNL under spinal anesthesia combined with sedation. Demographic characteristics illustrated in (Table 1). Mean preoperative Heart rate (HR) were comparable in both the groups (Table 2), group $\mathrm{KP} 77.11 \pm 8.23$ and group FP $75.62 \pm 6.73(\mathrm{P}=0.324)$. There was a significant increase in HR in KP group from $77.11 \pm 8.23$ to $84.38 \pm 7.96$ and there was decrease in FP group from $75.62 \pm 6.73$ to $66.27 \pm 7.21$ intraopertively $(\mathrm{P}=0.001)$. Mean postoperative pulse rate in KP group was $82.86 \pm 8.01$ compared to 67.45 \pm 7.14 in FP group $(\mathrm{P}=0.001)$. Systolic blood pressure (SBP) showed significant intra and post-operative increase in $\mathrm{KP}$ group compared to a decrease in $\mathrm{FP}$ group $(\mathrm{P}=0.001)$ (Figure 1). Concerning the diastolic blood pressure (DBP) showed intra and post-operative increase in KP group $(\mathrm{P} \leq 0.001)$ (Figure 2). Significant decrease in respiratory rate $(\mathrm{RR})$ and $\mathrm{SpO} 2$ was more pronounced in FP group during the intraoperative period $(\mathrm{P} \leq 0.001)$ (Table 3$)$, In reference to the side effects, no serious adverse effects were detected in both groups, $12 \mathrm{pa}$ tients developed apnea in FP group requiring minimal airway maneuvers like head tilt, jaw thrust and chin lift or nasopharyngeal airway insertion, none of the patients developed severe hypotension (Mean BP less than $50 \mathrm{mmHg}$ ) requiring aggressive management, Bradycardia (HR less than $50 \mathrm{~b} / \mathrm{min}$ ) noticed in 7 patients in the KP group required Atropine administration. Spo2 showed decrease postoperatively in FP $(\mathrm{P} \leq 0.001)$ only requiring oxygen supplementation (Figure 3). FP group spent more time in the recovery area (PACU) $6.38 \pm 1.02$ minutes compared to KP group $5.64 \pm 1.25$ min $(\mathrm{P} \leq 0.002)$, KP group showed significant analgesic effect after 1 and 3 hours from discharge $(P=0.001)$ with lower analgesic requirements (Table 4). 
Table 1. Demographic data.

\begin{tabular}{ccccc}
\hline Parameter & $\begin{array}{c}\text { Group I (KP) } \\
\mathbf{n}=\mathbf{5 0}\end{array}$ & $\begin{array}{c}\text { Group II (FP) } \\
\mathbf{n}=\mathbf{5 0}\end{array}$ & $\begin{array}{c}\text { Statistical } \\
\text { Test }\end{array}$ & P value \\
\hline Age & $33.87 \pm 14.80$ & $37.25 \pm 15.32$ & 1.12 & 0.26 \\
Sex (M:F) ratio & $26: 24(54 \%)$ & $28: 22(62 \%)$ & $0.161(\mathrm{chi})$ & 0.68 \\
Weight & $92.43 \pm 5.32$ & $94.32 \pm 5.94$ & 1.68 & 0.09 \\
\hline
\end{tabular}

Table 2. Mean Pulse difference in both groups.

\begin{tabular}{ccccccc}
\hline Parameter & \multicolumn{7}{c}{ KP } & \multicolumn{7}{c}{ FP } \\
\hline Pulse & Mean & SD & Mean & SD & T value & P value \\
Pre-operative & 77.11 & 8.23 & 75.62 & 6.73 & 0.99 & 0.324 \\
Intra-operative & 84.38 & 7.96 & 66.27 & 7.21 & 11.92 & 0.001 \\
Post-operative & 82.86 & 8.01 & 67.45 & 7.14 & 10.15 & 0.001 \\
\hline
\end{tabular}

Table 3. Mean respiratory difference in both groups.

\begin{tabular}{ccccccc}
\hline Parameter & \multicolumn{7}{c}{ KP } & \multicolumn{2}{c}{ FP } \\
RR & Mean & SD & Mean & SD & T value & P value \\
Pre-operative & 16.67 & 1.17 & 16.84 & 1.00 & 0.78 & 0.437 \\
Intra-operative & 16.12 & 1.02 & 8.21 & 1.32 & 16.57 & 0.000 \\
Post-operative & 16.53 & 1.19 & 16.54 & 0.96 & 0.89 & 0.534 \\
\hline
\end{tabular}

Table 4. Postoperative analgesia.

\begin{tabular}{cccc}
\hline Variable & KP & FP & P Value \\
\hline VAS 0 & 1 & 1 & 0.5 \\
VAS 1 & 1 & 3 & 0.001 \\
VAS 3 & 2 & 4 & 0.001 \\
VAS 6 & 2 & 3 & 0.002 \\
\hline
\end{tabular}

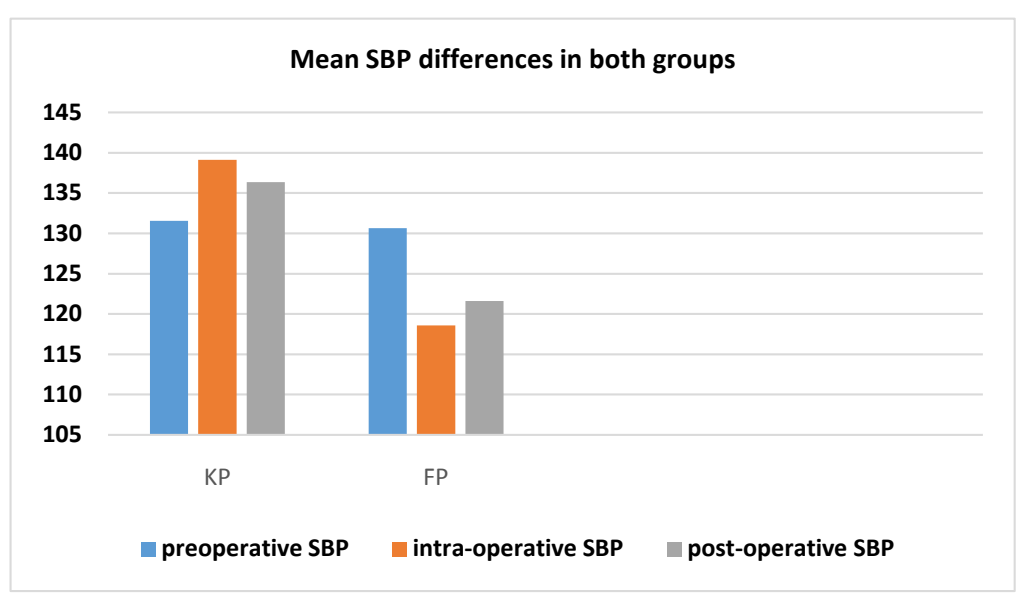

Figure 1. Mean SBP differences in both groups. Y Axis: Mean SBP in mmHg. 


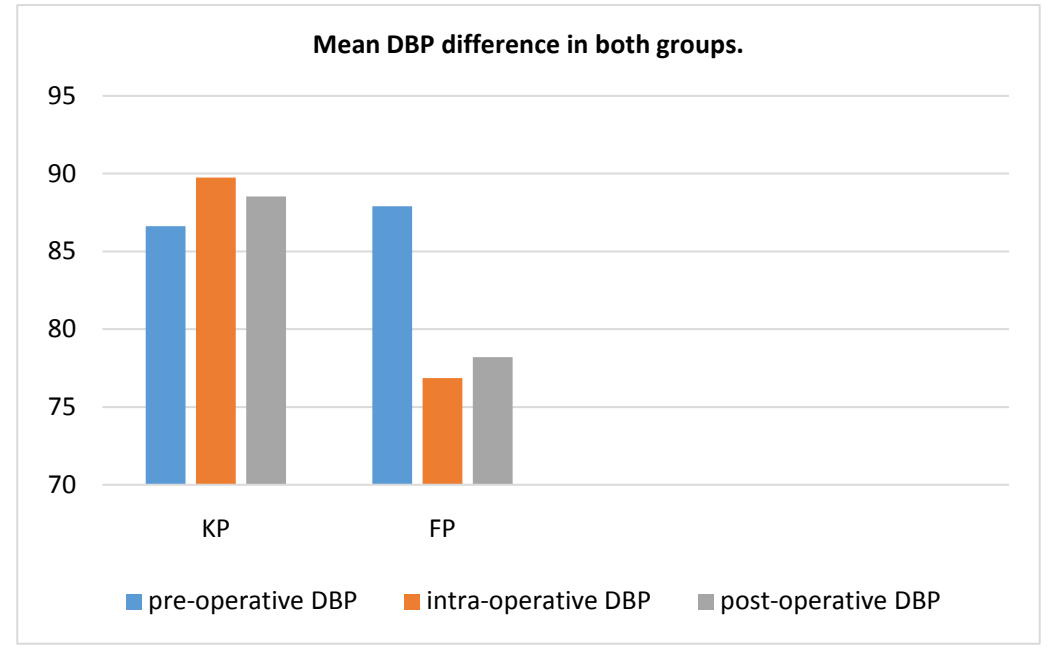

Figure 2. Mean DBP difference in both groups. Y Axis: Mean DBP in mmHg.

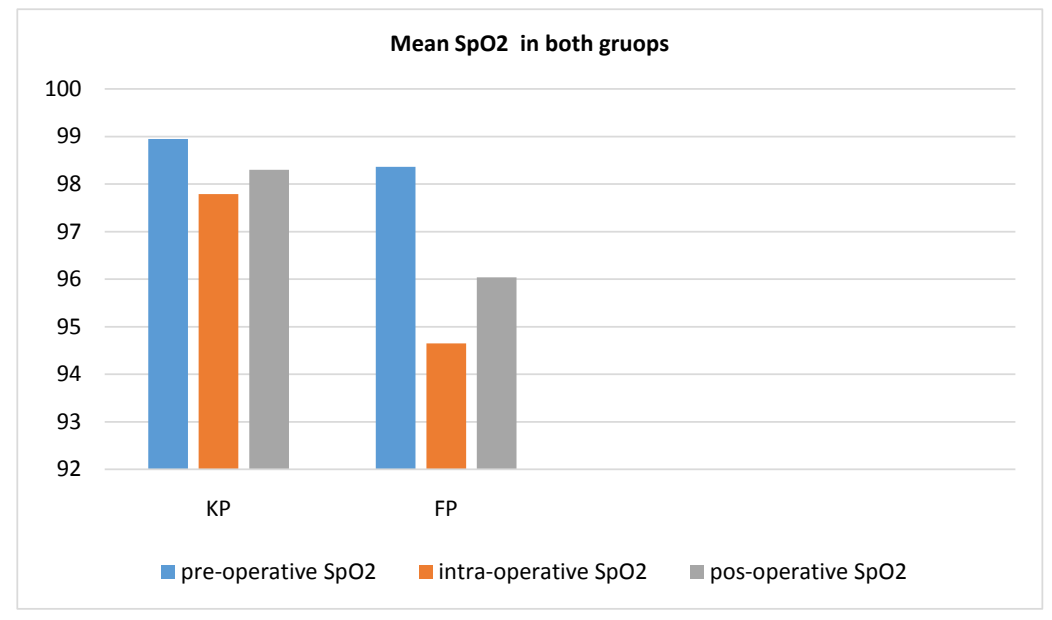

Figure 3. Mean SpO2 in both groups. Y Axis: Mean SpO2 \%.

\section{Discussion}

This study was conducted on 100 patients who were scheduled for elective PCNL under spinal anesthesia, patients were randomized in two groups KP group and FP group, Propofol lack analgesic properties and if used alone for anesthesia, the dose required will be large enough to cause cardio respiratory compromise, this necessitates concurrent administration of intravenous opioids and Paracetamol/NSAIDS as apart of multimodal balanced analgesia. Fentanyl is a commonly used opioid that provide rapid onset, short duration potent analgesia, useful during anesthetic induction. Using Ketamine and Propofol in combination allows sedation to be achieved at lower total doses of each drug [5], using a combination of Ketamine and Propofol with several ration of 2:1, 1:1 or 1:5 has been reported in several studies [14] [15], Fentanyl and PROP has been efficiently used in separate syringes by Bajwa and his colleagues [16] another study reported their mixing in the same syringe [17] in several procedures. In our study, we used a simple and easy method to maintain the level of sedation by adding 
increments of bolus doses were used to maintain a steady state level of sedation. This method is safe and applicable in our country due to the presence of several financial constraints limiting the presence of expensive monitors and disposables like bispectral index or electroencephalogram. Ketamine and Propofol in a ratio of 1:1 provided hemodynamic stability and are supported by the studies of Feky and colleagues [18]. Who compared the addition of PROP and Ketamine with PROP and fentanyl in pediatric patients who underwent upper gastrointestinal endoscopy, they concluded that the PK combination resulted in stable hemodynamics. There was pronounced bradycardia and hypotension in FP group. Our observations are supported by Khutia et al., who compared the infusion of both KP with FP in pediatric patients undergoing emergency short surgical procedures and found that HR and MAP were significantly decreased in the fentanyl group [14]. Bajwa et al. found slight respiratory depression in patients who received FP as compared to those who received KP [16]; this was due to the central depressant effect of fentanyl that may alter the respiratory rate and alveolar ventilation associated with narcotic analgesia that last longer than the analgesic effect. The peak respiratory depressant effect of a single intravenous dose of fentanyl is noted 5 to 15 minutes following injection. Fentanyl frequently slows the respiratory rate, but this effect is seldom noted for longer than 30 minutes regardless of the dose administered. Respiratory depression was not found in patients of KP group in our study. Our observations are supported by study of Nalini et al. who reported that none of the patients who received KP did not have any episodes of oxygen desaturation, airway obstruction or apnea, in contrast to patients who received FP, significant number had oxygen desaturation caused by airway obstruction [19]. The addition of low dose Ketamine to PROP improves ventilation and reduces the risk of respiratory depression. This may be due to effect of Ketamine induced sympathoadrenal activation [14]. To prevent Ketamine induced emergence reactions, pretreatment with benzodiazepines is commonly used. Likewise, the incidence of psychotomimetic responses was small when Ketamine was combined with PROP for general anesthesia or sedation [20]. In conjuction with our study as we used Ketamine with PROP and premedication of Midazolam decreasing the emergence reaction and vomiting of Ketamine. There was no complication like emergence reaction, agitation, increased oral secretions in our study and only one patient in FP group had nausea-vomiting. It seems use of routine premedication in our study helped us in prevention of these adverse effects. We used individual agents to address all parameters of balanced anesthesia technique and drugs to counter possible adverse effects of constituents of sedation solutions. Midazolam premedication to took care of anxiety, agitation and emergence reaction. Atropine controlled excessive bradycardia and salivation preventing laryngospasm and apnea while ondansetron premedication mitigated incidence of nausea and vomiting in our study. All patients were satisfied with their anesthesia experience. Urologists found their experience and working conditions convenient and excellent and they even demanded it for other short procedures that we usually used to do under regional 
anesthesia.

There is a limitation in our work, the personal variability between patients satisfaction and their confidence with the efficacy of sedative techniques in such procedures, in addition to limitations towards the surgical techniques and differences between surgeons.

\section{Conclusion}

From this study we concluded that various sedation techniques may be applicable for PCNL procedures with routine premedication, mixtures of fentanyl-Propofol or Ketamine-Propofol could be used, and KP has advantages of stable hemodynamics, prolonged analgesic response intra and post-operatively with no appreciable side effects and more effective method of sedative mixture.

\section{Conflict of Interest}

Nothing to disclose.

\section{Author's Contribution}

We hereby declare that the article has not been published or submitted to or accepted for publication in any form in any other journal. I vouch that the authorship of this manuscript will not be contested by anyone whose names are not listed. On acceptance the article will become the copyright of Journal. The manuscript has been read and approved by all the authors.

\section{References}

[1] Ramakumar, S. and Segura, J.W. (2000) Renal Calculi. Percutaneous Management. Urologic Clinics of North America, 27, 617-622. https://doi.org/10.1016/S0094-0143(05)70111-7

[2] Marcovich, R. and Smith, A.D. (2005) Percutaneous Renal Access: Tips and Tricks. BJU International, 95, 78-84. https://doi.org/10.1111/j.1464-410X.2005.05205.x

[3] Macario, A., Weinger, M., Carney, S. and Kim, A. (1999) Which Clinical Anaes-Thesia Outcomes Are Important to Avoid? The Perspective of Patients. Anesthesia \& Analgesia, 89, 652-658.

[4] Smith, I., Monk, T.G., White, P.F. and Ding, Y. (1994) Propofol Infusion during Regional Anaesthesia: Sedative, Amnestic, and Anxiolytic Proper-Ties. Anesthesia \& Analgesia, 79, 313-319. https://doi.org/10.1213/00000539-199408000-00020

[5] Willman, E.V. and Andolfatto, G. (2007) A Prospective Evaluation of "Ketofol" (Ketamine/Propofol Combination) for Procedural Sedation and Analgesia in the Emergency Department. Annals of Emergency Medicine, 49, 23-30. https://doi.org/10.1016/j.annemergmed.2006.08.002

[6] Doufas, A.G., Wadhwa, A., Shah, Y.M., Lin, C.M., Haugh, G.S. and Sessler, D.I. (2004) Block-Dependent Sedation during Epidural Anaesthesia Is Associated with Delayed Brainstem Conduction. British Journal of Anaesthesia, 93, 228-234. https://doi.org/10.1093/bja/aeh192

[7] Inagaki, Y., Mashimo, T., Kuzukawa, A., Tsuda, Y. and Yoshiya, I. (1994) Epidural Lidocaine Delays Arousal from Isoflurane Anaesthesia. Anesthesia \& Analgesia, 79, 
368-372. https://doi.org/10.1213/00000539-199408000-00030

[8] Schulz-Stubner, S. (2002) Clinical Hypnosis Instead of Drug-Based Sedation for Procedures under Regional Anesthesia. Regional Anesthesia and Pain Medicine, 27, 622-623. https://doi.org/10.1097/00115550-200211000-00018

[9] Kenny, G.N. (1996) Patient Sedation: Technical Problems and Developments. European Journal of Anaesthesiology, 13, 18-21. https://doi.org/10.1097/00003643-199607001-00005

[10] Taylor, E., Ghouri, A.F. and White, P.F. (1992) Midazolam in Combination with Propofol for Sedation during Local Anaesthesia. Journal of Clinical Anesthesia, 4, 213-216. https://doi.org/10.1016/0952-8180(92)90068-C

[11] Nakagawa, M., Mammoto, T., Sakai, T., Kishi, Y. and Mashimo, T. (2001) Premedication Modifies the Quality of Sedation with Propofol during Regional Anaesthesia. Canadian Journal of Anesthesia, 48, 284-287. https://doi.org/10.1007/BF03019760

[12] Corssen, G., Guttierrez, J., Reves, J.G. and Huber, F.C. (1972) Ketamine in Anesthetic Management of Asthmatic Patients. Anesthesia \& Analgesia, 51, 588-596. https://doi.org/10.1213/00000539-197207000-00027

[13] Ahmed, D.G., Hetta, D.F. and Abdelraouf, A.M.S. (2017) Percutaneous Nephrolithotomy under Thoracic Paravertebral Block: A Preliminary Report. Journal of Anesthesia and Clinical Research, 8, 720. https://doi.org/10.4172/2155-6148.1000720

[14] Khutia, S.K., Mandal, M.C., Das, S. and Basu, S.R. (2012) Intravenous Infusion of Ketamine-Propofol Can Be an Alternative to Intravenous Infusion of Fentanyl-Propofol for Deep Sedation and Analgesia in Paediatric Patients Undergoing Emergency Short Surgical Procedures. Indian Journal of Anaesthesia, 56, 145-150. https://doi.org/10.4103/0019-5049.96313

[15] Frizelle, H.P., Duranteau, J. and Samii, K. (1997) A Comparison of Propofol with Propofol Ketamine Combination for Sedation during Spinal Anaesthesia. Anesthesia \& Analgesia, 84, 1313-1322. https://doi.org/10.1213/00000539-199706000-00026

[16] Bajwa, S.S., Bajwa, S.K. and Kaur, J. (2010) Comparison of Two Drug Combinations in Total Intravenous Anaesthesia: Propofolketamine and Propofol-Fentanyl. Saudi Journal of Anaesthesia, 4, 72-79. https://doi.org/10.4103/1658-354X.65132

[17] Singh, R., Ghazanwy, M. and Vajifdar, H. (2013) A Randomized Controlled Trial to Compare Fentanyl-Propofol and Ketamine-Propofol Combination for Procedural Sedation and Analgesia in Laparoscopic Tubal Ligation. Saudi Journal of Anaesthesia, 7, 24-28. https://doi.org/10.4103/1658-354X.109801

[18] Feky, E.M. and Koptan, H.M. (2015) Midazolam, Ketamine, or Fentanyl Added to Propofol as Total Intravenous Anaesthesia in Skin Grafting after Burn in Paediatrics: A Comparative Study. Ain-Shams Journal of Anaesthesiology, 8, 160-165. https://doi.org/10.4103/1687-7934.156664

[19] Nalini, K.B., Cherian, A., Balachander, H. and Kumar, C.Y. (2014) Comparison of Propofol and Ketamine versus Propofol and Fentanyl for Puerperal Sterilization, a Randomized Clinical Trial. Journal of Clinical and Diagnostic Research, 8, GC01-04.

[20] Badrinath, S., Avramov, M.N., Shadrick, M., Witt, T.R. and Ivankovich, A.D. (2000) The Use of a Ketamine-Propofol Combination during Monitored Anesthesia Care. Anesthesia \& Analgesia, 90, 858-862. https://doi.org/10.1213/00000539-200004000-00016 